\title{
De que Conhecimento sobre Natureza da Ciência Estamos Falando?
}

\section{What Knowledge of the Nature of Science We Are Talking About?}

\author{
Paula Cristina Cardoso Mendonça' \\ 'Universidade Federal de Ouro Preto (UFOP), Departamento de Química, Ouro Preto, MG, Brasil. \\ E-mail: paulamendonca@ufop.edu.br
}

Resumo: Neste artigo apresentamos o que entendemos como um conhecimento amplo de Natureza da Ciência ( $\mathrm{NdC}$ ) levando em conta o objetivo do letramento científico para a Educação em Ciências. Esta discussão se mostra especialmente relevante porque há uma divergência de posicionamento na área. Podemos considerar como abordagem integrada de NdC para atingir tal finalidade: a análise pelos estudantes das práticas dos cientistas a partir do uso de estudos de caso da ciência, e a participação dos estudantes nas práticas científicas e a reflexão metacognitiva sobre os processos de justificação e validação do conhecimento. As duas situações são importantes porque capacitam a compreensão do trabalho dos cientistas em si, e o desenvolvimento do raciocínio científico ao aplicá-lo e analisá-lo em contexto. Este entendimento requer uma abordagem de $\mathrm{NdC}$ que não desvincula os processos científicos de seus produtos do conhecimento epistêmico envolvido na legitimação deles.

Palavras-chave: Natureza da ciência; Letramento; Educação científica.

\begin{abstract}
In this paper, we discuss what we can consider as a broad knowledge of Nature of Science (NOS), regarding the aim of scientific literacy for Science Education. This discussion is especially relevant since there is a divergence of positioning in the field. We can consider as an integrated approach of NOS to achieve this purpose: (i) students' analysis of scientists' practices from the use of science case studies; (ii) student participation in scientific practices and metacognitive reflection on the processes of justification and validation of knowledge. Both situations are important since they enable the understanding of the work of the scientists themselves; and the development of scientific reasoning by applying and analyzing it in context. Such understanding requires an approach of NOS that does not dissociate products and processes from epistemic knowledge involved in the legitimization of them.
\end{abstract}

Keywords: Nature of science; Literacy; Scientific education.

Recebido em: 10/03/2019

Aprovado em: 07/09/2019 


\section{Introdução}

Uma das metas primordiais da Educação Científica é o desenvolvimento do raciocínio científico e do pensamento crítico pelos alunos a partir de suas vivências de situações problemas no contexto de ensino. Exercer o pensamento crítico e as formas de raciocinar científicas podem ajudar os estudantes a investigar problemas, questionar e analisar de forma consciente e crítica os enunciados nos diferentes contextos e situações que vierem a lidar nas diversas demandas de atuação na sociedade (SASSERON, 2018). Frente a esta finalidade, destacamos a importância de que nos processos de ensino e aprendizagem, como destacado por Dushl (2008), o trabalho ocorra levando em consideração os três tipos de conhecimento: conceitual, epistêmico e social?

A discussão das grandes ideias das ciências, seus principais modelos, teorias e leis se torna relevante para o desenvolvimento conceitual dos estudantes, ampliando suas formas de ver e atuar no mundo a partir das lentes das ciências. Tais discussões deveriam ocorrer a partir da apresentação dos conceitos científicos em seus contextos de desenvolvimento, destacando-se as evidências que os fundamentam. Nesse sentido, o conhecimento epistêmico torna-se relevante, porque possibilita perceber como ocorrem os debates dos conceitos e ideias científicas ou o que conta nas tomadas de decisões nas comunidades científicas (SASSERON; DUSCHL, 2016), visto que sua ênfase está nos processos justificatórios do pensamento (KELLY, 2008). Por sua vez, o conhecimento social se torna importante para que a ênfase da pedagogia baseada na transmissão dos conhecimentos e na predominância do discurso do professor em sala de aula (com pouco espaço para questionamento, argumentação, respeito as ideias e discussão de posições alternativas) seja substituída pela pedagogia da construção e avaliação do conhecimento. Isto porque a autoridade epistêmica do conhecimento será distribuída no contexto de sala de aula a partir da negociação social do mesmo (DILLON; MANNING, 2010; KELLY, 2008; STROUPE, 2014).

Como consequência, para Kelly (2008), a aprendizagem de ciências requer a participação dos estudantes em práticas sociais relacionadas à produção, avaliação, comunicação e legitimação do conhecimento. O autor conceitua prática social como "aquela construída por uma série de ações, realizadas por membros de um grupo, baseadas em propósitos e expectativas comuns, e que compartilham valores culturais, ferramentas e significados" (KELLY, 2008, p. 102, tradução nossa). E quando tais padrões de ações estão dirigidos ao conhecimento podem ser chamados de epistêmicos.

$\mathrm{Na}$ visão de Osborne (2014), o conhecimento epistêmico está relacionado ao componente saber porque, isto é, o conhecimento de como os processos científicos justificam nossa crença nas afirmativas de conhecimento. Osborne (2014) nos diz que um crítico de música pode muito bem apreciá-la sem saber tocar um instrumento, da mesma forma, um crítico de arte pode criticá-la sem ser artista. Isto nos induz a pensar que não praticantes da ciência poderiam compreendê-la e serem críticos a respeito das afirmações na medida que conhecem como ocorrem os processos científicos.

\section{Apresentação do Problema}


No campo de Educação em Ciências, o termo Natureza da Ciência (aqui denominado $\mathrm{NdC}$ ) tem sido utilizado para fazer menção aos conhecimentos epistemológicos da ciência .que são potencialmente úteis aos estudantes da educação básica e professores desta área, de forma a caracterizar adequadamente as disciplinas da área de ciências da natureza. Esse construto híbrido apropria-se de reflexões das disciplinas metacientíficas, preponderantemente: filosofia, história e sociologia da ciência (IZQUIERDO-AYMERICH; ADÚRIZ-BRAVO, 2003).

Muitas pesquisas sobre $\mathrm{NdC}$ têm investigado a concepção de ciência de professores e estudantes de ciências da natureza de diversos níveis de escolaridade sobre a ciência que é praticada pelos cientistas profissionais, classificando-as em ingênuas ou adequadas a partir de princípios determinados a priori sobre o que conta como um conhecimento esclarecido de ciências, como, por exemplo, Lederman (2007), Mccomas e Olson (1998), e Schwartz, Lederman e Abd-El-Khalick (2012).

Outras pesquisas estão mais preocupadas com as visões sobre ciência dos professores e estudantes ao se engajarem efetivamente em investigações ou práticas que visam a construção e avaliação do conhecimento, como, por exemplo, modelagem e argumentação (JIMÉNEZ-ALEIXANDRE; CRUJEIRAS, 2017; KELLY, 2008; OSBORNE, 2016; ÖSTMAN; WICKMAN, 2014; SANDOVAL, 2005, 2014). Os pesquisadores estão preocupados em entender como a partir de tarefas específicas socialmente contextualizadas o conhecimento epistêmico se desenvolve. Eles partem do pressuposto que o aprendizado dos processos cognitivos, sociais, epistêmicos e materiais da ciência são fundamentais para que o aluno compreenda a natureza da ciência. Torna-se necessário esclarecer que o termo epistêmico é distinto de epistemologia, apesar de ambos guardarem relação com o conhecimento. Epistêmico está relacionado à justificativa do conhecimento em si. Por exemplo, afirmar que uma alegação se torna mais confiável a partir do uso de evidências. Enquanto epistemologia, originalmente, se refere ao ramo da filosofia que estuda o conhecimento (teoria do conhecimento). Essa área se preocupa com questões relacionadas a distinção entre aquilo que é ou não é conhecimento (SANDOVAL, 2014).

Então, por um lado, várias pesquisas sobre $\mathrm{NdC}$ investigam a concepção de ciência sobre o que o cientista faz e sobre como ocorre a produção do conhecimento na ciência, por exemplo, a partir da análise dos estudos de caso da ciência (casos históricos ou contemporâneos da ciência); enquanto outras estão mais relacionadas com aquilo que os estudantes aprendem sobre ciência ao se envolverem em um contexto das práticas ou investigações científicas. O termo práticas científicas pode ser considerado mais abrangente do que investigação científica, uma vez que o último, geralmente, está associado a trabalhos práticos ou tarefas de laboratório, enquanto o primeiro seria mais abrangente em função de englobar uma série de ações, tarefas e performances análogas às científicas, tais como: desenvolver e usar modelos; argumentar a partir das evidências; elaborar explicações; planejar e conduzir investigações etc.

Esse artigo tem como o objetivo contribuir com esclarecimentos frente ao entendimento do que seria um conhecimento amplo de $\mathrm{NdC}$ e coerente com os objetivos atuais da Educação Científica. O artigo tem caráter teórico e visa esclarecer esses aspectos com base em nossas reflexões e a partir de uma revisão crítica da literatura. 


\section{Abordagem Consensual de Natureza da Ciência}

Existe uma discussão na área de Educação em Ciências sobre a (im)possibilidade de se definir o que seja ciência para fins pedagógicos (ABD-EL-KHALICK, 2012; ALLCHIN, 2011; HODSON, 2014; IRZIK; NOLA, 2011; JUSTI; ERDURAN, 2015; MCCOMAS, 2008; NIELSEN, 2012; OSBORNE et al., 2003; VAN DICK, 2011), considerando que não há objeção de posição quanto à impossibilidade de definição da ciência nos campos disciplinares específicos, como a filosofia (ALTERS, 1997; EFLIN; GLENNAN; REISCH, 1999). Uma posição essencialista da ciência implica em defini-la a partir de uma lista de critérios característicos que são utilizados para discriminar uma disciplina como científica. Por outro lado, a posição não essencialista defende que a ciência não pode ser plenamente descrita, que não há uma única forma de caracterizá-la que seja capaz de levar em conta a heterogeneidade das disciplinas científicas, mesmo para fins pedagógicos (EFLIN; GLENNAN; REISCH, 1999).

As propostas essencialistas que mais se destacam na área de Educação em Ciências são a visão consensual ou os sete princípios, como, por exemplo, em Abd-El-Khalick (2005, 2012), Lederman $(2006,2007)$ e Lederman et al. (2002). Considera-se de grande relevância para a área dos sete princípios, o pioneirismo da proposta de Osborne et al. (2003) por ter sido a primeira baseada em consulta aos especialistas. Para mais detalhes sugerimos consultar Acevedo-Díaz e García-Carmona (2016).

Lederman (2006) especifica que apesar de reconhecer que entre especialistas que estudam o assunto não há consenso sobre a demarcação do que é ciência, ele defende que produzir uma lista de aspectos consensuais é relevante para fins educativos.

Lederman (2006) justifica a elaboração da lista para estudantes da educação básica a partir dos seguintes critérios: aspectos compreensíveis aos estudantes; características consensuais entre especialistas; utilidade para os cidadãos. Com base em tais critérios, o pesquisador nos apresenta os aspectos que visam caracterizar a ciência e que deveriam fazer parte do currículo de estudantes da educação básica: conhecimento científico tem bases empíricas (baseia-se em experimentos e observações); é confiável, porém susceptível a falhas e/ou provisório (sujeito a mudanças, não sendo uma verdade absoluta); é produto da imaginação e criatividade humana; é guiado por teorias e subjetivo (influenciando pelos conhecimentos prévios, crenças, experiências e expectativas dos cientistas); é culturalmente e socialmente imbricado (é influenciado pelos contextos sociais e culturais). Além disso, ele destaca que observações são diferentes de inferências; teorias e leis são categorias distintas do conhecimento; não existe um único método científico que seja capaz de produzir conhecimento confiável.

Em termos de abordagem no ensino, o pesquisador é favorável ao uso de atividades investigativas para introdução de $\mathrm{NdC}$, de modo que o docente deve auxiliar os estudantes a refletir de modo explícito sobre os aspectos sobre ciência da lista consensual relacionados a investigação. Ele argumenta que o modo implícito não é o mais adequado, porque de forma isolada o estudante pode não ser capaz de relacionar os pontos chaves da investigação com a natureza do conhecimento científico. Todavia, o autor deixa claro que investigação é distinto de $\mathrm{NdC}$, porque o último se refere a epistemologia da ciência, a ciência como forma de conhecimento, os valores e crenças inerentes ao conhecimento científico e ao seu desenvolvimento. 
Outra abordagem essencialista na área de Educação em Ciências é aquela desenvolvida por Osborne et al. (2003). Os pesquisadores realizaram um estudo empírico ${ }^{1}$ com diversos especialistas no assunto (professores de ciências, filósofos, sociólogos, divulgadores da ciência e cientistas), ao passo que a maioria das abordagens consensuais foi baseada na análise de documentos oficiais e o foco era mais restrito aos aspectos filosóficos e sociológicos do conhecimento (ver por exemplo, Mccomas e Olson, 1998). Os itens aos quais se obteve elevado índice de consenso entre os especialistas foram: (i) método científico $e$ testes críticos (inclui a ideia de que o questionamento contínuo e o teste experimental das afirmativas científicas são aspectos centrais no desenvolvimento da pesquisa científica); (ii) hipóteses e previsão (a ideia de que formular hipóteses e propor previsões em relação a elas é algo essencial à ciência); (iii) análises e interpretações de dados (dados não falam por si só, são passíveis de múltiplas interpretações); (iv) ciência e certeza (inclui a ideia de que o conhecimento científico é provisório); (v) diversidade do pensamento científico (existe uma variedade de caminhos e métodos de pesquisa que são escolhidos em função dos problemas); (vi) criatividade (a pesquisa científica requer muita criatividade, como na criação e teste de modelos para explicar fenômenos); (vii) desenvolvimento histórico do conhecimento científico (a história da ciência pode auxiliar na percepção da ciência como um empreendimento humano, evidenciando como a ciência afeta e é afetada pelas demandas sociais e expectativas); (viii) cooperação e colaboração no desenvolvimento do conhecimento científico (a ideia de que a ciência é uma atividade que se desenvolve em grupos de pesquisa e que envolve mecanismos como revisão entre os pares e diferentes intercâmbios).

Quanto à abordagem no ensino, Osborne et al. (2003) concluíram que não é viável discutir cada aspecto de $\mathrm{NdC}$ de forma fragmentada, isto é, os pontos convergentes devem ser tratados de forma integrada, sendo que as investigações científicas e os estudos de caso seguidos de reflexão sobre a prática científica potencializam este tipo de discussão.

As abordagens consensuais de NdC propostas a partir de distintos critérios podem ser consideradas parciais frente ao caráter dinâmico da ciência. Portanto, tratam-se de escolhas, nas quais os critérios empregados dependem fundamentalmente da forma como as metaciências são priorizadas (ACEVEDO-DÍAZ et al., 2007). NdC tem tradicionalmente sido vista de um ponto de vista mais restrito à epistemologia (LEDERMAN, 2007). Outras concepções se relacionam a diversas questões entrelaçadas com ciência, como tecnologia e sociedade, sociologia interna da ciência, imagem da ciência, atitudes da ciência escolar, práticas científicas etc. (ACEVEDO-DÍAZ; GARCÍA-CARMONA, 2016; ALLCHIN, 2017; HODSON, 2014). Nesse sentido, há um debate intenso entre os pesquisadores da área sobre os problemas de uma posição essencialista no ensino de ciências, principalmente filiada às questões mais epistemológicas, o que tem levado a divulgação de posições alternativas.

\section{Abordagem Alternativa para o Ensino de Natureza da Ciência}

\footnotetext{
${ }^{1}$ Os autores recorreram à metodologia Delphi, que pode ser entendida como uma forma de decisão grupal a partir da avaliação de opiniões sem interações face a face, que se dá a partir do julgamento de tópicos particulares coletados previamente via questionário. Inicialmente, os participantes escreveram de forma geral (e com exemplos e indicativos de contextos) o que deveria ser falado aos estudantes da educação básica sobre natureza do conhecimento científico, métodos da ciência e os aspectos sociais da prática científica. As respostas foram incialmente categorizadas e posteriormente analisadas em duas rodadas pelos especialistas a partir do uso de escala Likert. Em seguida, foram analisadas pelos pesquisadores utilizando-se parâmetros como média, moda e desvio padrão da média para se verificar o nível de consenso.
} 
$\mathrm{Na}$ literatura são apresentados dois argumentos centrais contrários à posição essencialista de inserção de $\mathrm{NdC}$ no ensino de ciências. O primeiro argumento se relaciona a falha em representar a heterogeneidade da ciência, pois corre-se o risco de ao simplificar a ciência a partir de uma lista de tópicos fundamentais fazer generalizações que não são aplicáveis a todos os campos científicos (ALLCHIN, 2017; IRZIK; NOLA, 2014; WONG; HODSON, 2010). Existem diferenças significativas entre os campos disciplinares da ciência, como os tipos de questões que se propõem a investigar, as técnicas e metodologias utilizadas nas investigações, o tipo de evidência a que têm acesso, como utilizam, e com quão extensão a experimentação, a forma como os dados são coletados, a forma como as conclusões são avaliadase a maneira como são reportadas. Por exemplo, as geociências são fundamentalmente interpretativas e históricas ao invés de experimentais, e objetivam explicar muitos eventos do passado a partir da proposição de modelos causais. Mesmo dentro da própria disciplina os modos de investigação e objetivos podem ser bem distintos. Por exemplo, algumas áreas da química orgânica têm um forte viés experimental com o objetivo de sintetize de novas moléculas e substâncias e análise de várias de suas propriedades a partir de métodos físicos e químicos. Por outro lado, a química teórica utiliza de técnicas computacionais e modelos matemáticos na tentativa de previsão de novas moléculas e seus comportamentos.

O segundo argumento está relacionado a dicotomia dos tópicos da lista consensual ou a expressão de características fora de um contexto (ELBY; HAMMER, 2001; IRZIK; NOLA, 2014). Por exemplo, ao se afirmar que a ciência tem caráter subjetivo, poderia implicar em negar a objetividade que deveria ser também inerente ao fazer científico, uma vez que cientistas devem tentar evitar fazer conclusões precipitadas e enviesar a análise. Todavia, pelo fato da ciência ser um empreendimento humano, o cientista tem expectativas, crenças e outros tipos variados de influências que interferem no seu trabalho.

Elby e Hammer (2001), ao analisarem as abordagens consensuais, advertem que julgar que uma posição pode ser mais filosoficamente sofistica da quea outra éingênuo se não julgada dentro de um contexto. Como exemplo, quanto ao critério provisoriedade do conhecimento, os autores demostram que tal concepção é problemática, porque é equivocado afirmar que todos os conhecimentos da ciência têm o mesmo grau de provisoriedade que outros. Existem conhecimentos que estão muito bem estabelecidos nas ciências (por exemplo, as Leis de Newton) e com os quais os cientistas trabalham sem controvérsias. Para os autores, o uso do critério provisoriedade na ciência no ensino pode ser improdutivo porque pode levar a uma visão relativista ingênua da ciência, na qual o conhecimento científico é visto como uma construção arbitrária humana, sem os constrangimentos impostos pela natureza. Os autores concluem que uma visão sofisticada da ciência não incorpora generalizações apriorísticas.

Como pode-se perceber pelos argumentos aqui expostos, é problemático caracterizar a ciência de forma homogênea em função de sua natureza multifacetada e complexa. Além disso, as características que a definem só fazem sentido quando atreladas aos contextos (JUSTI; ERDURAN, 2015; WONG; HODSON, 2010).

Tais críticas contribuíram para alguns pesquisadores se posicionarem contrariamente as abordagens essencialistas e defenderem o ensino de NdC contextualizado (ALLCHIN, 2011; ALLCHIN; ANDERSEN; NIELSEN, 2014; HODSON, 2014; JUSTI; MENDONÇA, 2016). Ou seja, a partir da análise de casos da ciência que será possível levantar os aspectos que a caracterizam. Isto significa que um determinado contexto poderá ser mais propício para tratar de aspectos sociológicos e econômicos da ciência, enquanto outros poderão favorecer um entendimento mais profundo dos aspectos cognitivos da ciência, como os tipos de questionamentos, 
raciocínio científico, métodos científicos etc. (JUSTI; ERDURAN, 2015).

Com relação à abordagem contextualizada, pesquisadores (por exemplo ALLCHIN; ANDERSEN; NIELSEN, 2014; MOURA; GUERRA, 2016; WONG; HODSON, 2010) apresentam dois tipos de contextos propícios para desenvolvimento de visões de NdC: atividades investigativas ou práticas da ciência e estudos de caso (contemporâneos e históricos). Os casos contemporâneos fazem referência a casos da ciência que ainda são controversos e cujos temas estão em discussão na comunidade científica. Os estudantes podem tornar-se aptos a analisar aspectos como: incerteza, provisoriedade, subjetividade, múltiplas perspectivas, o papel do financiamento, interesses políticos e o contexto social que influencia e é influenciado pela ciência (ALLCHIN; ANDERSEN; NIELSEN, 2014). No entanto, casos contemporâneos não favorecem aos estudantes compreender como ocorreu a resolução das controvérsias científicas, o que pode ser contemplado ao se trabalhar com os casos históricos.

Os casos históricos podem ser compreendidos como casos da ciência em que os conhecimentos científicos já foram consolidados, não sendo mais alvo atual de disputa entre os cientistas, mas que já o foram no passado. Eles podem ilustrar o papel do debate e crítica na ciência, as parcialidades teóricas, culturais e cognitivas, as motivações, as chances, a colaboração, conexões interdisciplinares, experiência e credibilidade, conflitos de interesse etc. (ALLCHIN; ANDERSEN; NIELSEN, 2014). Entretanto, os estudantes devem ser de fato envolvidos no contexto histórico, eles não devem apenas observar ou comentar sobre histórias. Por exemplo, devem ser envolvidos em dramatizações históricas, júri simulado, experimentos históricos, entre outros recursos (JUSTI; MENDONÇA, 2016). Os estudantes devem ter a perspectiva de ciência em construção de uma forma contextualizada, de modo a ter conhecimento das ideias, incertezas e expectativas com as quais os cientistas do passado tiveram que lidar à luz do contexto da época em questão (MOURA; GUERRA, 2016).

As atividades investigativas e práticas científicas no ensino de ciências devem relacionar-se com as ações, ferramentas, práticas e interações discursivas da ciência, e assim favorecer o seu entendimento epistêmico e processual. Os estudantes podem adquirir noção de como as afirmativas científicas são construídas e avaliadas a partir da análise das evidências ( ALLCHIN; ANDERSEN; NIELSEN, 2014).

Alguns pesquisadores (ALLCHIN, 2011, 2013; MATTHEWS, 1998; SMITH; SCHARMAN, 1998), por discutirem a complexidade de se polarizar o que é ou não é ciência, argumentam em favor de uma abordagem que capacite os estudantes a entender quais questões podem ser ou não respondidas pela ciência ou quais critérios levam uma questão a ser mais ou menos científica que outra (SMITH; SCHARMAN, 1998). Ou ainda quais critérios podem levar uma afirmativa do conhecimento ser confiável ou não (ALLCHIN, 2011, 2013).

Nesse sentido, Allchin (2011), ao argumentar em favor de uma abordagem contextualizada da ciência, é favorável a evitar o uso de listas engessadas que a caracterizam. Assim, nos apresenta uma rubrica para análise das dimensões da confiabilidade das afirmativas científicas (relacionado à observação, métodos de investigação, instrumentação, padrões de raciocínio, dimensões históricas, dimensões humanas, interações entre cientistas, dimensões socioculturais, economia e/ou financiamento e comunicação do conhecimento). Para ilustrar essas ideias o autor apresenta um estudo de caso que relata a história de uma mulher de 40 anos de idade preocupada com a possibilidade de ter um câncer de mama e que planeja fazer uma mamografia, apesar dos seus receios em relação à radiação excessiva. No entanto, a mulher recebe a informação de que uma força tarefa nacional americana aconselha que se espere até os 50 anos de idade para realizar o exame, sendo esta informação confirmada 
na revista Women's Health. Porém, a mesma mulher conheceu outra que faleceu aos 43 anos de idade com câncer de mama (ALLCHIN, 2011). A partir dessas informações, os estudantes devem avaliar as evidências que são disponibilizadas em artigos científicos e revistas destinadas ao público feminino, para tomarem a decisão se a mulher deveria fazer a mamografia ou esperar até completar 50 anos. Com tal abordagem, o pesquisador argumenta a favor do desenvolvimento de uma compreensão funcional da ciência, que estaria atrelada ao letramento científico. Isto porque Allchin (2011) parte do princípio de que os sujeitos devem desenvolver uma compreensão sobre como a ciência funciona com o objetivo de interpretar a confiabilidade das afirmativas científicas em tomadas de decisão.

Segundo o autor, para se atingir a finalidade de formar cidadãos críticos torna-se relevante um ensino sobre ciência que os capacite a questionar as conclusões científicas e que os leve a entender como uma afirmativa de conhecimento é estabelecida, se mantêm ou se modifica ao longo do tempo. Mesmo afirmativas confiáveis podem aparecer com qualificadores e ressalvas, de forma que seus significados se tornam claros apenas quando é possível entender os vários métodos utilizados para promover confiabilidade, bem como suas limitações (ALLCHIN, 2017).

Ainda, de acordo com Allchin (2011), apenas o conhecimento declarativo dos princípios da lista consensual (ABD-EL-KHALICK, 2012; LEDERMAN, 2007; LEDERMAN et al., 2002) não garante que tais objetivos ocorram. Isto porque o aluno afirmar, por exemplo, que inferências são distintas de observações, não garante que ele saiba usar esse conhecimento de forma procedimental ao analisar um enunciando e perceber se há dados que o corroboram em termos de confiabilidade e suficiência para que seja possível estabelecer certas conclusões.

Kelly (2008) também se posiciona de forma similar, pois para o autor, trabalhar com uma visão prescritiva de epistemologia é pouco relevante para os objetivos da Educação em Ciências. Quanto à questão da epistemologia da ciência, Kelly (2008, p. 291, tradução nossa) deixa claro que:

Minha visão é de que Natureza da Ciência (ou melhor a Natureza das Ciências)
não deveria ser limitada as afirmativas sobre ciência e nem a pedagogia
deveria ser tão limitada a reflexão explícita de tais pontos. Em alguns casos,
o processo reflexivo explícito de se pensar sobre NdC pode fazer sentido,
como no caso da formação do professor, mas em outros, em que os objetivos
pedagógicos se diferem, apenas a explicitação de tais princípios é limitada.

Nessa direção, Allchin $(2011,2013)$ afirma que entender NdC de forma ampla inclui não apenas a síntese de elementos centrais de $\mathrm{NdC}$, mas também os processos e produtos científicos. Para o autor, ao se utilizar estudos de caso da ciência e a reflexão e explicitação de como nós sabemos, ao se discutir como a ciência trabalha e quais métodos, processos e valores são utilizados nas escolhas e julgamentos, será possível possibilitar um entendimento mais amplo sobre ciência, contribuindo de fato para o letramento científico. Para Allchin (2011, 2013), os alunos precisam saber responder questões do tipo: Como avaliar determinada informação sabendo-se que há discordância entre pesquisadores? Como avaliar se as informações são confiáveis e verossímeis? Que tipos de condições justificam mudanças de consenso científico? 
A discussão dos parágrafos anteriores, relacionado à mudança de foco no ensino de NdC - de uma lista de princípios desprovida de contexto - para a análise das práticas científicas, das questões científicas e da credibilidade das fontes, informações e afirmativas científicas - nos conduz para uma controvérsia sobre a inserção de $\mathrm{NdC}$ no currículo de ciências: a distinção entre epistemologia e práticas científicas ou investigação científica ${ }^{1}$ do que $\mathrm{NdC}$, enquanto eles se propõe a fazer uma clara distinção entre natureza da investigação científica e natureza da ciência ou natureza do conhecimento científico (epistemologia). Mais recentemente, Lederman (2018) continuou apontando como problemática a não distinção entre NdC (epistemologia) de investigação ou práticas científicas, a partir de uma análise do documento americano mais recente ${ }^{2}$ (USA, 2012). Em tal documento, $\mathrm{NdC}^{3}$ é apresentado como um subconjunto das práticas científicas e conceitos transversais, e considera-se que a sua aprendizagem se dará mediante conexões que poderão ser estabelecidas durante a vivência e discussão das práticas.

\section{Análise Crítica das Abordagens}

A literatura aqui apresentada nos indica que há posicionamentos divergentes no campo da Educação em Ciências sobre as abordagens empregadas visando uma compreensão ampla de NdC com a finalidade do letramento científico e a formação de cidadãos críticos. Há uma divergência quanto o uso de listas que caracterizariam a ciência a priori, como também uma crítica em se igualar o entendimento de $\mathrm{NdC}$ a compreensão das investigações ou práticas científicas. Em outros termos, o conhecimento epistêmico desenvolvido pelos estudantes no contexto de desenvolvimento de práticas científicas conta como um conhecimento de $\mathrm{NdC}$ ? Ou como trabalhar no ensino de ciências de forma a capacitar o desenvolvimento de um conhecimento amplo de $\mathrm{NdC}$ ? Considerando tais questionamentos, a seguir são apresentadas considerações relevantes para nossa reflexão.

Jiménez-Aleixandre e Crujeiras (2017) consideram que no campo das didáticas das ciências a epistemologia está mais ligada a examinar o desenvolvimento do conhecimento, a natureza das evidências, a eleição das teorias e a estrutura do conhecimento disciplinar. Para as autoras, a epistemologia no campo da didática das ciências está preocupada em responder a questões como: por que sabemos o que sabemos? o que sabemos exatamente? Nesse sentido, elas consideram que a vivência reflexiva das práticas constitui uma forma eficaz para monitorar as concepções e crenças dos alunos sobre a ciência.

Para Moura e Guerra (2016, p. 751, tradução nossa), a ênfase em práticas científicas pode ser uma perspectiva bem sucedida para o debate sobre as questões relacionadas a $\mathrm{NdC}$, uma vez que pode direcionar a caminhos alternativos que seguem as caracterizações consensuais do conhecimento científico. Contudo, "[...] mudar o foco da essência da ciência

\footnotetext{
${ }^{1} \mathrm{O}$ que eles chamam de NOSI em língua inglesa (Nature of Scientific Investigation).

${ }^{2} \mathrm{O}$ documento americano apresenta como um dos objetivos para a Educação Científica dos estudantes da educação básica o trabalho com oito práticas científicas em especial, a saber: fazer questões, desenvolver e usar modelos, elaborar explicações, produzir argumentos baseados em evidências, planejar e construir investigações, coletar e interpretar dados, usar pensamento matemático e computacional.

${ }^{3} \mathrm{Alguns}$ enunciados de $\mathrm{NdC}$ são apresentados relacionados às práticas científicas: as investigações científicas usam uma variedade de métodos científicos; conhecimento científico é empiricamente fundamentado; conhecimento científico é susceptível a revisão com base nas novas evidências; os modelos, leis e teorias explicam os fenômenos naturais; a ciência é uma forma de saber; a ciência é um esforço humano; a ciência aborda perguntas do mundo natural e material e supõe uma ordem e consistência dos sistemas naturais.
} 
para as práticas científicas coloca desafios em relação ao desenvolvimento e implementação de abordagens históricas dentro da sala de aula".

Moura e Guerra (2016, p. 753, tradução nossa) também advertem que

\begin{abstract}
[...] abordar as práticas reais dos cientistas não garantem, por si só, sua percepção epistemológica, nem possibilitam a compreensão da ciência como construções sócio-históricas e culturais. Assim, apoiamos a relevância de uma visão externa para práticas científicas que permitam analisar como a ciência funciona e o efeito de seus produtos na sociedade, além da dependência dos produtos criados para as demandas da sociedade. Nesse sentido, é essencial explorar o contexto histórico da produção científica, bem como suas relações com outras produções culturais que fazem parte desse contexto.
\end{abstract}

De forma similar, para Allchin, Andersen e Nielsen (2014), um problema de focar o ensino de $\mathrm{NdC}$ em práticas científicas é a construção de uma visão epistemológica da ciência escolar, em que os estudantes podem vir a pensar que a prática científica se realiza de forma idêntica aquela da prática escolar. Estudantes podem não transferir suas vivências da ciência no contexto escolar para outros contextos reais, de modo que se torna amplamente necessário que professores e materiais instrucionais discutam os conhecimentos relacionados a NdC gerados a partir de investigações realizadas pelos estudantes para contextos mais reais. Em complemento, existem vários contextos científicos nos quais características centrais da ciência, tais como parcialidades culturais, conflitos de interesse econômico, contingência histórica, credibilidade, expertise etc. que não podem ser facilmente modeladas em sala de aula a partir da abordagem das práticas científicas (ALLCHIN; ANDERSEN; NIELSEN, 2014).

Tomando por base as posições apresentadas nos parágrafos anteriores, podemos dizer que analisar e explorar as práticas científicas em situações educacionais sem considerar adequadamente a historicidade de tais práticas acarretaria em problemas relacionados a uma concepção limitada de $\mathrm{NdC}$, porque não contextualiza adequadamente o conhecimento no âmbito dos aspectos externalistas à ciência.

Por outro lado, uma forma viável de avaliar o entendimento de NdC seria a partir dos questionamentos em contextos específicos e da avaliação as respostas das tarefas efetuadas pelos alunos, uma vez que o conhecimento meramente declarado de aspectos que caracterizam a ciência não indica uma compreensão funcional de $\mathrm{NdC}$, isto é, que capacite compreender os processos de tomada de decisão na ciência. Nesse sentido, torna-se necessário avaliar os entendimentos processuais e epistêmicos da ciência de forma conjugada (ALLCHIN, 2011; OSBORNE, 2014, 2016). Isto porque há uma convergência de posição de que o entendimento e a avaliação sobre ciência deve ocorrer em contextos autênticos, isto é, aqueles fundamentados na análise crítica, problematizadora e reflexiva da prática científica, seja a partir da análise de estudos de caso ou na participação reflexiva e contextualizada historicamente e socialmente das práticas científicas escolares.

Para se atingir contextos autênticos de ensino de $\mathrm{NdC}$, dois pontos de partida são importantes: (a) os objetivos da educação em ciências são distintos dos objetivos da ciência, pois no primeiro caso, a preocupação está no entendimento de um conhecimento já estabelecido, e, no segundo caso, a produção do próprio conhecimento; (b) a ciência não deve ser encarada na educação em ciências como um modo de conhecimento superior aos demais, mas como uma forma de saber, cujos tipos de questões, métodos, processos, justificativas e raciocínios variam entre os seus diferentes campos 
disciplinares (e dentro das próprias disciplinas) em função de suas idiossincrasias.

Levando em conta tais pontos, para uma compreensão mais ampla de $\mathrm{NdC}$ no contexto do ensino de ciências torna-se necessário: (1) discutir o que torna a ciência distinta de outros empreendimentos humanos a partir do conhecimento dos modos de raciocínios e das diferentes ferramentais representacionais, intelectuais e discursivas empregadas neste campo do saber que conduzem os cientistas a legitimarem leis, teorias, modelos e afirmativas do conhecimento; (2) utilizar o raciocínio científico e suas ferramentas intelectuais, representacionais e discursivas para questionar as fontes de informação, avaliar os critérios utilizados pelos especialistas para fazer determinadas considerações, identificar argumentos de autoridade e distinguir afirmativas baseadas em evidência de mera opinião (ALLCHIN, 2011; HODSON, 2014; SANDOVAL, 2014; SASSERON; DUSCHL, 2016; SMITH; SCHARMAN, 1998).

Pensando-se nos objetivos (1) e (2), eles são capazes de anteder a compreensão da ciência praticada pelos cientistas profissionais e os aspectos epistêmicos da ciência vinculados às práticas científicas. Porém, isto requer uma abordagem de $\mathrm{NdC}$ que não desvincula processos de produtos do conhecimento epistêmico envolvido na legitimações deles.

Isto nos conduz a pensar que relacionar NdC estritamente à epistemologia, mais no campo da esfera filosófica, isto é, os valores e normas relacionados à produção do conhecimento, tal como defendido por Lederman e seus colaboradores (ABD-EL-KHALICK, 2005; LEDERMAN, 2007, 2018; SCHWARTZ; LEDERMAN; ADB-EL-KHALICK, 2012), não é satisfatório aos objetivos aqui discutidos. Isto porque para saber distinguir a ciência dos outros campos dos saberes torna-se necessário pensar nos processos ou práticas da ciência.

Aspectos presentes na abordagem consensual tais como imaginação, criatividade, ciência como empreendimento humano, fazem parte de outros modos de produção do saber, assim como de outras atividades humanas, como a arte e a estética, a antroposofia e a religião. Por outro lado, o entendimento do papel dos processos comunicativos da ciência (uma prática científica) auxiliaria mais a entender a particularidade da linguagem científica. Nielsen (2012) nos esclarece que realizar uma distinção entre conhecimento científico e outras formas de conhecimento pode ser em função das diferenças entre os códigos da ciência e outras formas de codificação; e ao mesmo tempo, a identificação e operacionalização dos códigos da ciência. A linguagem científica permite a transformação da experiência em significado, a ciência sistematicamente faz uso do poder transformativo de certos elementos linguísticos na fabricação de novas estruturas de significado, alguns dos quais perdem a ligação com a própria experiência

Conclui-se, portanto, que torna-se inviável pensar em como poderiam ser atingidos os objetivos (1) e (2) para o ensino de NdC quando o pensamos estritamente associados à epistemologia tal como Lederman et al. (2012). Uma forma riquíssima de compreensão da ciência é justamente a compreensão de suas práticas de justificação: de como cientistas conseguem transformar dados em padrões e modelos, de como as explicações se tornam sustentadas por evidências e como elas sustentarão novas previsões, de como representações conseguem falar pelos cientistas e viajar no tempo e no espaço guardando e desempacotando vários significados etc. Como Osborne (2014) nos informa, as práticas da ciência incluem as dimensões: fazer ciência, ler ciência, escrever ciência, representar ciência, falar ciência. Logo, o entendimento de NdC deveria perpassar todas essas esferas - algo que a abordagem consensual não faz, porque além das características não atenderem a todas estas esferas, parte do princípio que práticas não contam como conhecimento de $\mathrm{NdC}$. 


\section{Considerações Finais}

A ciência produz cada vez mais conhecimento e aumenta a nossa compreensão sobre o mundo, consequentemente nossa visão sobre NdC deveria evoluir com tempo. Nesse artigo fizemos um esforço de mostrar certas convergências sobre os estudos epistemológicos na Educação em Ciências frente ao avanço do conhecimento nesse campo de pesquisa e os objetivos do letramento científico.

Sobre a questão de pesquisa investigada podemos considerar que compreender amplamente $\mathrm{NdC}$ inclui entender a prática do cientista por meio da análise dos estudos de caso da ciência, como também a participação dos estudantes nas práticas científicas e a reflexão sobre eleas relacionando-se ao trabalho dos cientistas. As vivências das duas situações são importantes porque capacitam compreender o trabalho dos cientistas em si, bem como desenvolver o raciocínio científico a partir da compreensão de como aplicálo e analisá-lo em contexto. Cabe destacar que não basta os alunos serem envolvidos nas práticas científicas para que eles aprendam sobre ciência, há a necessidade de um ensino explícito sobre as práticas, isto é, de envolver os alunos na reflexão sobre as práticas, o que estaria relacionado ao como sabemos o que sabemos e o por que acreditamos no que sabemos. A perspectiva das práticas contribui para que o aluno aprenda sobre a ciência, porque além dele vivenciar um ambiente similar, no qual ele participa de algumas práticas, ele tem oportunidade de se engajar em um processo metacognitivo sobre como ele fez para atribuir credibilidade ao conhecimento e a relação disto com o modo como a ciência opera.

Podemos considerar também que há uma convergência de pensamento em torno de abandonar o foco nos conhecimentos declarativos sobre ciência e sua respectiva avaliação em termos do que os alunos ou professores sabem de ciência frente aos tópicos das abordagens consensuais. Muitas pesquisas que apenas classificam a visão dos estudantes e professores em determinadas categorias (LEDERMAN, 2007) falham em dar esclarecimentos sobre como levar os sujeitos a desenvolver noções mais sofisticadas de ciência a partir de seus conhecimentos prévios ( EFLIN; GLENNAN; REISCH, 1999).

Avaliar crenças sobre ciência não é o ponto central do que se espera de um entendimento sofisticado de $\mathrm{NdC}$, pois a independência intelectual é um dos objetivos da Educação Científica. Isto porque educar não visa somente os sujeitos adquirirem as crenças corretas, mas ter as adequadas razões para essas crenças. A independência intelectual ou pensamento crítico requer dos sujeitos argumentar, analisar posições contrárias e refutá-las, e ainda, ser capaz de criticar as próprias posições (SMITH; SCHARMAN, 1998).

A partir dessa mudança de foco no que ensinar, a avaliação de $\mathrm{NdC}$ se dará fundamentalmente em termos do entendimento dos tipos de perguntas lançadas e investigadas pela ciência, seus modos de investigação, o que torna um conhecimento mais ou menos científico, os tipos de raciocínio e as diversas normas, padrões, ações e críticas envolvidas nos processos de produção, comunicação, avaliação e legitimação do conhecimento. Ou seja, é a partir do entendimento conjugado de produtos, processos e aspectos epistêmicos envolvidos na ciência que estudantes compreenderão a sua natureza.

É importante que os professores de ciências tenham em mente que a abordagem dos processos, produtos e aspectos epistêmicos da ciência deve acontecer de forma articulada e integrada. A avaliação de $\mathrm{NdC}$ em termos de tais componentes do conhecimento configura-se como autêntica aos objetivos do letramento científico (ALLCHIN, 2011). Como 
exemplos do que se espera de conhecimento de NdC: entender porque uma explicação científica é eleita frente a outras alternativas; quais dados se transformam em evidência para respaldar uma afirmativa; como cientistas entram em determinado consenso sobre certos conhecimentos; que aspectos contam na tomada de decisão nos casos da ciência; como a divulgação do conhecimento é importante para que os enunciados se tornem fatos; como ampliar a objetividade científica a partir da crítica pelos pares etc.

A proposta de análise das dimensões da confiabilidade das afirmativas científicas elaborada por Allchin (2011) apresenta-se coerente como perspectiva de avaliação dos conhecimentos de $\mathrm{NdC}$ nesta perspectiva. O autor nos apresenta 41 itens relacionados a análise epistêmica das afirmativas da ciência, que podem ser levados em consideração ao se analisar reportagens científicas extraídas da mídia (como os exames para prevenção da câncer de mama, autismo, vacinação etc.). Certamente torna-se complicado dar conta de abordar todos os itens no ensino, mas alguns aspectos importantes da proposta podem ser selecionados para dar foco a alguns aspectos de NdC salientes aos casos sob análise. Cabe destacar que não se trata de uma proposta que visa homogeneizar nossa compreensão do que seja ciência. Podemos pensar na rubrica mais como de caráter ilustrativo do que exaustivo. Além disso, o autor é favorável ao uso da rubrica como critérios de avaliação associados a instrução baseada em casos históricos da ciência, questões mais contemporâneas da ciência, geralmente, a partir dos casos sociocientíficos, e da vivência reflexiva das práticas científicas.

As discussões aqui traçadas nos direcionam a pensar na formação de professores de ciências. Apenas o domínio declarado de $\mathrm{NdC}$ pelos professores de ciências não é condição capaz de levá-los a adotar estratégias eficientes do seu ensino em sala de aula, pois isto depende também dos modelos instrucionais em que o professor foi educado. $\mathrm{Na}$ formação de professores observamos um predomínio de práticas de ensino fundamentadas na transmissão de um corpus de conhecimento reificado, no qual são ignoradas as bases epistêmicas do conhecimento e a natureza da própria disciplina (BARTHOLOMEW; OSBORNE; RATCLIFFE, 2004). O ensino efetivo de NdC também depende das oportunidades que o professor teve para desenvolver e aplicar o conhecimento da formação inicial em contextos da prática de ensino (BARTHOLOMEW; OSBORNE; RATCLIFFE, 2004).

Em virtude de tais problemas, é amplamente desejável que a formação de professores de ciências também trabalhe com propostas contextualizadas, fundamentadas em estudos de caso e práticas científicas, visando o ensino de $\mathrm{NdC}$ na dimensão do conhecimento funcional. Isto porque consideramos amplamente desejável que os professores tenham experiências de articular teoria e prática pensando-se no repertório docente para o ensino, pois como esperar que os professores ensinem sobre NdC a partir da pedagogia de construção e avaliação do conhecimento se forem instruídos a partir da pedagogia da transmissão dos conhecimentos? (TARDIF, 2002).

Não estamos desconsiderando a importância dos professores de ciências terem um aprofundamento teórico mais robusto, ou seja, eles devem conhecer em algum grau de profundidade as literaturas que embasam as propostas pedagógicas. Porém, não julgamos que o caminho sejam cursos de $\mathrm{NdC}$ desvinculados da prática pedagógica, porque não seriam suficientes para os professores reconhecerem os recursos e estratégias potencias para o ensino de NdC. 


\section{Agradecimentos}

Agradeço ao Departamento de Química da Universidade Federal de Ouro Preto pela liberação para capacitação para estudos no ano de 2018. Agradeço a professora Stefannie de Sá Ibraim, do Departamento de Química da Universidade de Brasília, e a aluna de doutorado em Educação do Programa de Pós-graduação em Educação da Universidade Federal de Ouro Preto, Tatiana Costa Ramos, pelos apontamentos na primeira versão do manuscrito.

\section{Referências}

GABD-EL-KHALICK, F. Developing deeper understandings of nature of science: the impact of a philosophy of science course on preservice science teachers' views and instructional planning. International Journal of Science Education, Abingdon, v. 27, n. 1, p. 15-42, 2005.

ABD-EL-KHALICK, F. Examining the sources for our understanding about science: enduring conflations and critical issues in research on nature of science in science education. International Journal of Science Education, Abingdon, v. 34, n. 3, p. 353-374, 2012.

ACEVEDO-DÍAZ, J. A.; GARCÍA-CARMONA, A. Algo antiguo, algo nuevo, algo prestado: tendencias sobre la naturaleza de la ciencia en la educación científica. Revista Eureka sobre Enseñanza y Divulgación de las Ciencias, Cádiz, v. 13, n. 1, p. 3-19, 2016.

ACEVEDO-DÍAZ, J. A.; VÁZQUEZ-ALONSO, A.; MANASSERO MAS, M. A.; ACEVEDO-ROMERO, P. Consensos sobre la naturaleza de la ciencia: fundamentos de una investigación empírica. Revista Eureka sobre Enseñanza y Divulgación de las Ciencias, Cádiz, v. 4, n. 1, p. 42-66, 2007.

ALLCHIN, D. Beyond the consensus view: whole science. Canadian Journal of Science and Mathematics and Technology Education, Heidelberg, v. 17, n. 1, p. 18-26, 2017.

ALLCHIN, D. Evaluating knowledge of the nature of (whole) science. Science Education, Hoboken, v. 95, n. 3, p. 518-542, 2011. DOI: https://doi.org/10.1002/sce.20432

ALLCHIN, D. Teaching the nature of science: perspectives \& resources. Saint Paul, MN: SHiPS Education Press, 2013.

ALLCHIN, D.; ANDERSEN, H. M.; NIELSEN, K. H. Complementary approaches to teaching nature of science: integrating student inquiry, historical cases, and contemporary cases in classroom practice. Science Education, Hoboken, v. 98, n. 3, p. 461-486, 2014. DOI: https://doi.org/10.1002/sce.21111

ALTERS, B. J. Whose nature of science? Journal of Research in Science Teaching, Hoboken, v. 34, p. 39$55,1997$.

BARTHOLOMEW, H.; OSBORNE, J.; RATCLIFFE, M. Teaching students "ideas-about-science": five dimensions of effective practice. Science Education, Hoboken, v. 88, n. 5, p. 655-682, 2004.

DILLON, J.; MANNING, A. Science teachers, science teaching: issues and challenge. In: OSBORNE, J.; DILLON, J. (ed.). Good practice in science teaching: what research has to say. Berkshide: McGraw Hill, 2010. p.6-19.

DUSCHL, R. A. Science education in three-part harmony: balancing conceptual, epistemic, and social learning goals. Review of Research in Education, Thousand Oaks, v. 32, n. 1, p. 268-291, 2008. DOI: https://doi.org/10.3102/0091732X07309371 
EFLIN, J. T.; GLENNAN, S.; REISCH, G. The nature of science: a perspective from the philosophy of science. Journal of Research in Science Teaching, Hoboken, v. 36, n. 1, p. 107-116, 1999.

ELBY, A.; HAMMER, D. On the substance of a sophisticated epistemology. Science Education, Hoboken, v. 85, n. 5, p. 554-567, 2001. DOI: https://doi.org/10.1002/sce.1023

HODSON, D. Nature of science in science curriculum: origin, development, impliciations and shifiting emphasis. In: MATTHEWS, M. (ed.). International handbook of research in history, philosophy and science teaching. Dordrecht: Springer, 2014. p. 911-970.

IRZIK, G.; NOLA, R. A family resemblance approach to the nature of science for science education. Science \& Education, Dordrecht, v. 20, n. 7-8, p. 59-607, 2011.

IRZIK, G.; NOLA, R. New directions for nature of science research. In: MATTHEWS, M. (ed.). International handbook of research in history, philosophy and science teaching. Dordrecht: Springer, 2014. p. 999-1021.

IZQUIERDO-AYMERICH, M.; ADÚRIZ-BRAVO, A. Epistemological foundations of school science. Science \& Education, Dordrecht, v. 12, n. 1, p. 27-43, 2003.

JIMÉNEZ-ALEIXANDRE, M. P.; CRUJEIRAS, B. Epistemic pratices and scientific pratices in science education. In: TABER, K.; AKPAN, B. (ed.). Science education: an international course companion. Rotterdam: Sense Pub., 2017. p. 69-80.

JUSTI, R.; ERDURAN, S. Caracterizing nature of science: a supporting model for teachers. In: HPST biennal conference, 13., 2015, Rio de Janeiro. Proceedings [...]. Disponível em: http://www.ihpst.net/ content.aspx?page_id=22\&club_id=360747\&module_id=187166. Acesso em: 10 mar. 2020.

JUSTI, R.; MENDONÇA, P. C. C. Discussion of the controversy concerning a historical event among pre-service teachers. Science \& Education, Dordrecht, v. 25, n. 1, p. 795-822, 2016.

KELLY, G. Inquiry, actitivity and epistemic practice. In: DUSCHL, R.; GRANDY, R. (ed.). Teaching scientific inquiry: recommendations for research and implementation. Rotterdam: Sense Pub., 2008. p. 288-291.

LEDERMAN, N. G. Nature of science: past, present, and future. In: ABELL, S. K.; LEDERMAN, N. G. (ed.). Handbook of research on science education. Mahwah: Lawrence Erlbaum, 2007. p. 831-880.

LEDERMAN, N. G. La siempre cambiante contextualización de la natureza da ciencia: documentos recientes sobre la reforma de la educación científica en los Estados Unidos y su impacto en el logro de la alfabetización cientifíca. Enseñanza de las Ciencias, Barcelona, v. 36, n. 2, p. 5-22, 2018.

LEDERMAN, N. G. Syntax of nature of science within inquiry and science instruction. In: FLICK, L. B.; LEDERMAN, N. G. (ed.). Scientific inquiry and nature of science. Dordrecht: Springer, 2006. p. 301-317.

LEDERMAN, N. G.; ABD-EL-KHALICK, F.; BELL, R. L.; SCHWARTZ, R. Views of nature of science questionnaire: toward valid and meaningful assessment of learner's conceptions of nature of science. Journal of Research in Science Teaching, Hoboken, v. 39, n. 6, p. 497-521, 2002.

MATTHEWS, M. R. In defense of modest goals when teaching about the nature of science. Journal of Research in Science Teaching, Hoboken, v. 35, n. 2, p. 161-174, 1998.

McCOMAS, W. F. Seeking historical examples to illustratte key aspects of the nature of science. Science \& Education, Hoboken, v. 17, n. 2-3, p. 249-263, 2008.

McCOMAS, W. F.; OLSON, J. K. The nature of science in international science standards documents. In: McCOMAS, W. F. (ed.). The nature of science in science education: rationalities and strategies. Dordrecht: Kluwer, 1998. p. 44-51. 
MOURA, C.; GUERRA, A. Cultural history of science: a possible path for discussing scientific practices in science teaching? Revista Brasileira de Pesquisa em Educação em Ciências, Rio de Janeiro, v. 16, n. 3, p. 749-771, 2016.

NIELSEN, K. H. Scientific communication and the nature of science. Science \& Education, Hoboken, v. 22, n. 9, p. 2067-2086, 2012. DOI: https://doi.org/10.1007/s11191-012-9475-3

OSBORNE, J. Teaching scientific practices: meeting the challenge of change. Journal of Science Teacher Education, Abingdon, v. 25, n. 2, p. 177-196, 2014.

OSBORNE, J. Defining a knwoledge base for reasoning in science: the role of procedural and epistemic knowledge. In: DUSCHL, R.; BISMARCK, A. S. (ed.). Reconceptualizing STEM education: the central role of practice. New York: Routledge 2016. p. 215-231.

OSBORNE, J. et al. What "ideas-about-science" should be taugth in school science?: a delphi study of the expert community. Journal of Research in Science Teaching, Hoboken, v. 40, n. 7, p. 692-720, 2003.

ÖSTMAN, L.; WICKMAN, P.-O. A pragmatic approach on epistemology, teaching, and learning. Science Education, Abingdon, v. 98, n. 3, p. 375-382, 2014.

SANDOVAL, W. Science education's need for a theory epsitemological development. Science Education, Abingdon, v. 98, n. 3, p. 383-387, 2014.

SANDOVAL, W. Understanding student's pratical epistemologies and their influence on learning through inquiry. Science Education, Abingdon, v. 89, n. 4, p. 634-656, 2005.

SASSERON, L. H. Ensino de ciências por investigação e o desenvolvimento de práticas: uma mirada para a base nacional comum. Revista Brasileira de Pesquisa em Educação em Ciências, Rio de Janeiro, v. 18, p. 1-25, 2018.

SASSERON, L. H.; DUSCHL, R. Ensino de ciências e práticas epistêmicas: o papel do professor e o engajamento dos estudantes. Investigações em Ensino de Ciências, Porto Alegrre, v. 21, n. 2, p. 52-67, 2016.

SCHWARTZ, R. S.; LEDERMAN, N. G.; ABD-EL-KHALICK, F. A series of misrepresantions: a responde to Allchin's whole approach to assessing nature of science understandings. Science Education, Abingdon, v. 96, n. 4, p. 685-692, 2012.

SMITH, M. U.; SCHARMAN, L. C. Defining versus describing the nature of science: a pragmatic analysis for classroom teachers and science educatiors. Science Education, Abingdon, v. 85, n. 4, p. 493-504, 1998.

STROUPE, D. Examining classroom science practice communities: how teachers and students negotiate epistemic agency and learn science-as-practice. Science Education, Abington, v. 98, n. 3, p. 487-516, 2014.

TARDIF, M. Saberes docentes e formação profissional. Petrópolis: Vozes, 2002.

USA. National Research Council of the National Academies. A framework for K-12 science education: practices, crosscutting concepts, and core ideas. Washington: National Academy Press, 2012.

VAN DICK, E. M. Portraying real science in science communication. Science Education, Abingdon, v. 95, n. 6, p. 1086-1100, 2011. DOI: https://doi.org/10.1002/sce.20458

WONG, S. L.; HODSON, D. More from the horse's mouth: what scientists say about science as a social practice. International Journal of Science Education, Abingdon, v. 32, n. 11, p. 1431-1463, 2010. 